\title{
Functional representation for the grand partition function of a multicomponent system of charged particles: Correlation functions of the reference system
}

\author{
O.V.Patsahan, I.M.Mryglod \\ Institute for Condensed Matter Physics of the National Academy of Sciences of Ukraine, \\ 1 Svientsitskii Str., 79011 Lviv, Ukraine
}

Received February 27, 2006, in final form June 29, 2006

\begin{abstract}
Based on the method of collective variables (CV) with a reference system, the exact expression for the functional of the grand partition function of a $m$-component ionic model with charge and size asymmetry is found. Particular attention is paid to the $n$th particle correlation functions of the reference system which is presented as a $m$-component system of "colour" hard spheres of the same diameter. A two-component model is considered in more detail. In this case the recurrence formulas for the correlation functions are found. A general case of a $m$-component inhomogeneous system of the "colour" hard spheres is also analysed.
\end{abstract}

Key words: functional methods, reference system, collective variables method, asymmetric ionic model, "colour" hard sphere system

PACS: $05.70 . F h, 05.70 . J k, 02.70 . R r, 64.70 . F x$

\section{Introduction}

In recent years much attention has been focused on an issue of the phase transitions in multicomponent fluid mixtures, especially in ionic fluids. In spite of significant progress in this field, such systems are far from being completely understood. The investigation of complex models is of great importance in understanding the nature of critical and phase behavior of real ionic fluids which demonstrate both the charge and size asymmetry. The powerful tools for the study of multicomponent continuous systems are those based on the functional methods. One of such approaches is the method of collective variables (CVs). The method, proposed initially in the 1950s [1-3] for the description of the classical charged many particle systems and developed later for the needs of the phase transition theory [4-7], was in fact one of the first successful attempts to attack the problems of statistical physics using the functional integral representation. Nearly at the same time other functional approaches based on the Stratonovich-Hubbard transformation [8,9] were originated. As was shown recently [10] both groups of theories are in fact in close relation.

The CV method is based on: (i) the concept of collective coordinates being appropriate for the physics of the system considered (see, for instance, [11]) and (ii) the integral identity allowing to derive an exact functional representation for the configurational Boltzmann factor. Being applied to the continuous system the CV method uses the idea of the reference system (RS), one of the basic ideas of the liquid state theory [12]. The idea consists in the splitting of an interparticle interaction potential into two parts

$$
u_{\alpha \beta}(r)=\phi_{\alpha \beta}^{0}(r)+\phi_{\alpha \beta}(r),
$$

where $\phi_{\alpha \beta}^{0}(r)$ is a potential of a short-range repulsion which describes the mutual impenetrability of the particles, while $\phi_{\alpha \beta}(r)$, on the contrary, mainly describes the behaviour at moderate and large distances. The equilibrium properties of the system interacting via the potential $\phi_{\alpha \beta}^{0}(r)$ are assumed to be known. Therefore, this system can be regarded as the "reference" system. Within the framework of the $\mathrm{CV}$ method the interaction connected with potential $\phi_{\alpha \beta}(r)$ is described in 
the phase space of CVs. The fluid of hard spheres is most frequently used as the RS in the liquid state theory since its thermodynamic and structural properties are well known. It is worth noting that the concept of the RS was also applied to the study of quasispin systems with short- and long-range interactions [13].

In this paper we derive the functional representation for the grand canonical partition function of a multicomponent fluid which includes both short-range and long-range interactions. A particular attention is focused on an issue of the correlation functions of the RS of a charge asymmetric continuous binary model of an ionic fluid. The paper is organized as follows. In section 1 we obtain the exact expression for the functional of the grand partition function of a multicomponent mixture. The case of an asymmetric ionic model is considered in more detail. Section 2 is devoted to the study of the correlation functions of a $m$-component hard sphere system of the same diameter (system of "colour" hard spheres). This system forms a basis for further applications of standard methods in the study of many particle interacting systems. The recurrence formulas for the correlation functions of the two-component system of "colour" hard spheres are found here.

\section{Method}

\subsection{General relations for a $m$-component system}

Let us consider a classical $m$-component system consisting of $N$ particles among which there exist $N_{1}$ particles of species $1, N_{2}$ particles of species $2, \ldots$ and $N_{m}$ particles of species $m$. The pair interaction potential is assumed to be of the form:

$$
U_{\alpha \beta}(r)=\phi_{\alpha \beta}^{\mathrm{HS}}(r)+\phi_{\alpha \beta}^{\mathrm{C}}(r)+\phi_{\alpha \beta}^{\mathrm{SR}}(r),
$$

where $\phi_{\alpha \beta}^{\mathrm{HS}}(r)$ is the interaction potential between the two additive hard spheres of diameters $\sigma_{\alpha \alpha}$ and $\sigma_{\beta \beta}$. We call the $m$-component hard sphere system a reference system $(\mathrm{RS}) . \phi_{\alpha \beta}^{\mathrm{C}}(r)$ is the Coulomb potential. The solution is made of both positive and negative ions so that electroneutrality is satisfied, $\sum_{\alpha=1}^{m} q_{\alpha} c_{\alpha}=0$, and $c_{\alpha}$ is concentration of the species $\alpha$. The ions of the $\alpha$ th species are characterized by their hard sphere diameter $\sigma_{\alpha \alpha}$ and their electrostatic charge $q_{\alpha}$. Here we consider the case when the hard spheres differ little in their diameters. Then we can present the potential of short-range interaction $\phi_{\alpha \beta}^{\mathrm{SR}}(r)$ as a sum of the two terms: $\phi_{\alpha \beta}^{\mathrm{SR}}(r)=\phi_{\alpha \beta}^{R}(r)+\phi_{\alpha \beta}^{A}(r)$, where $\phi_{\alpha \beta}^{R}(r)$ is used to mimic the soft core asymmetric repulsive interaction, $\phi_{\alpha \beta}^{R}(r)$ is assumed to have a Fourier transform. $\phi_{\alpha \beta}^{A}(r)$ describes a van der Waals like attraction. The above assumption about hard sphere diameters allows us to employ hereafter a one-component hard sphere system as the RS for the study of a multicomponent mixture.

We consider the grand partition function (GPF) of the system which can be presented in the form:

$$
\Xi\left[\nu_{\alpha}\right]=\sum_{N_{1}, N_{2}, \ldots, N_{m}} \prod_{\alpha=1}^{m} \frac{\exp \left(\nu_{\alpha} N_{\alpha}\right)}{N_{\alpha} !} \int(\mathrm{d} \Gamma) \exp \left[-\frac{\beta}{2} \sum_{\alpha, \beta} \sum_{i j} U_{\alpha \beta}\left(r_{i j}\right)\right] .
$$

Here the following notations are used: $\nu_{\alpha}=\beta \mu_{\alpha}+\ln \left[\left(2 \pi m_{\alpha} \beta^{-1}\right)^{3 / 2} / h^{3}\right]$ is the dimensionless chemical potential, $\mu_{\alpha}$ is the chemical potential of the $\alpha$ th species, $\beta$ is the reciprocal temperature, $(\mathrm{d} \Gamma)$ is the element of the configurational space of $N$ particles.

Let us introduce operators

$$
\hat{\rho}_{\mathbf{k}, \alpha}=\sum_{i} \exp \left(-\mathrm{i} \mathbf{k r}_{i}^{\alpha}\right)
$$

which are the Fourier transforms of the microscopic number density of the species $\alpha$. In this case we can present the part of the Boltzmann factor in (2) which does not include the RS interaction in the form:

$$
\exp \left[-\frac{\beta}{2} \sum_{\alpha, \beta} \sum_{i, j}\left(U_{\alpha \beta}\left(r_{i j}\right)-\phi_{\alpha \beta}^{\mathrm{HS}}\left(r_{i j}\right)\right)\right]=\exp \left[-\frac{1}{2} \sum_{\alpha, \beta} \sum_{\mathbf{k}} \tilde{\Phi}_{\alpha \beta}(k)\left(\hat{\rho}_{\mathbf{k}, \alpha} \hat{\rho}_{-\mathbf{k}, \beta}-N_{\alpha} \delta_{\alpha \beta}\right)\right]
$$


where

$$
\tilde{\Phi}_{\alpha \beta}(k)=\tilde{\Phi}_{\alpha \beta}^{\mathrm{C}}(k)+\tilde{\Phi}_{\alpha \beta}^{\mathrm{SR}}(k)
$$

and we use the notations $\tilde{\Phi}_{\alpha \beta}^{X \ldots}(k)=\frac{\beta}{V} \tilde{\phi}_{\alpha \beta}^{X \ldots}(k), \tilde{\phi}_{\alpha \beta}^{X \ldots}(k)$ is the Fourier transform of the corresponding interaction potential.

In order to introduce the collective variables $(\mathrm{CVs})$ we use the identity

$$
\exp \left[-\frac{1}{2} \sum_{\alpha, \beta} \sum_{\mathbf{k}} \tilde{\Phi}_{\alpha \beta}(k) \hat{\rho}_{\mathbf{k}, \alpha} \hat{\rho}_{-\mathbf{k}, \beta}\right]=\int(\mathrm{d} \rho) \prod_{\alpha} \delta_{\mathcal{F}}\left[\rho_{\mathbf{k}, \alpha}-\hat{\rho}_{\mathbf{k}, \alpha}\right] \exp \left[-\frac{1}{2} \sum_{\alpha, \beta} \sum_{\mathbf{k}} \tilde{\Phi}_{\alpha \beta} \rho_{\mathbf{k}, \alpha} \rho_{-\mathbf{k}, \beta}\right],
$$

where $\delta_{\mathcal{F}}\left[\rho_{\mathbf{k}, \alpha}-\hat{\rho}_{\mathbf{k}, \alpha}\right]$ denotes the functional delta function

$$
\delta_{\mathcal{F}}\left[\rho_{\mathbf{k}, \alpha}-\hat{\rho}_{\mathbf{k}, \alpha}\right] \equiv \int(\mathrm{d} \omega) \exp \left[\mathrm{i} \sum_{\mathbf{k}} \omega_{\mathbf{k}}\left(\rho_{\mathbf{k}, \alpha}-\hat{\rho}_{\mathbf{k}, \alpha}\right)\right],
$$

$\rho_{\mathbf{k}, \alpha}=\rho_{\mathbf{k}, \alpha}^{c}-\mathrm{i} \rho_{\mathbf{k}, \alpha}^{s}$ is the CV which describes the value of the $\mathbf{k}$-th fluctuation mode of the density of the $\alpha$ th species particles. $(\mathrm{d} \rho)$ is a volume element of the $\mathrm{CV}$ phase space:

$$
(\mathrm{d} \rho)=\prod_{\alpha} \mathrm{d} \rho_{0, \alpha} \prod_{\mathbf{k} \neq 0}^{\prime} \mathrm{d} \rho_{\mathbf{k}, \alpha}^{c} \mathrm{~d} \rho_{\mathbf{k}, \alpha}^{s} .
$$

The prime means that the product over $\mathbf{k}$ is performed in the upper semi-space. The indices $c$ and $s$ denote the real and imaginary parts of $\rho_{\mathbf{k}, \alpha}$.

Taking into account (4)-(5), we can present (2) in the form:

$$
\Xi\left[\nu_{\alpha}\right]=\int(\mathrm{d} \rho)(\mathrm{d} \omega) \exp \left(-\mathcal{H}\left[\nu_{\alpha}, \rho_{\alpha}, \omega_{\alpha}\right]\right),
$$

where

$$
\mathcal{H}\left[\nu_{\alpha}, \rho_{\alpha}, \omega_{\alpha}\right]=\frac{1}{2} \sum_{\alpha, \beta} \sum_{\mathbf{k}} \tilde{\Phi}_{\alpha \beta}(k) \rho_{\mathbf{k}, \alpha} \rho_{-\mathbf{k}, \beta}-\mathrm{i} \sum_{\alpha} \sum_{\mathbf{k}} \omega_{\mathbf{k}, \alpha} \rho_{\mathbf{k}, \alpha}-\ln \Xi_{H S}\left[\bar{\nu}_{\alpha} ;-\mathrm{i} \omega_{\alpha}\right] .
$$

$\Xi_{H S}\left[\bar{\nu}_{\alpha} ;-\mathrm{i} \omega_{\alpha}\right]$ is the GPF of a $m$-component system of the bare hard spheres with the renormalized chemical potential $\bar{\nu}_{\alpha}$ in the presence of the local field $\psi_{\alpha}\left(r_{i}\right)$

$$
\Xi_{H S}[\ldots]=\sum_{N_{1}, N_{2}, \ldots, N_{m}} \prod_{\alpha=1}^{m} \frac{\exp \left(\bar{\nu}_{\alpha} N_{\alpha}\right)}{N_{\alpha} !} \int(\mathrm{d} \Gamma) \exp \left[-\frac{\beta}{2} \sum_{\alpha, \beta} \sum_{i, j} \phi_{\alpha \beta}^{\mathrm{HS}}\left(r_{i j}\right)+\sum_{\alpha} \sum_{i}^{N_{\alpha}} \psi_{\alpha}\left(r_{i}\right)\right],
$$

where

$$
\begin{gathered}
\bar{\nu}_{\alpha}=\nu_{\alpha}+\frac{1}{2} \sum_{\mathbf{k}} \tilde{\Phi}_{\alpha \alpha}(k), \\
\psi_{\alpha}\left(r_{i}\right)=-\mathrm{i} \omega_{\alpha}\left(r_{i}\right) .
\end{gathered}
$$

Mean-field approximation. The mean-field approximation of functional (6) is defined by

$$
\Xi_{M F}\left[\nu_{\alpha}\right]=\exp \left(-\mathcal{H}\left[\nu_{\alpha}, \bar{\rho}_{\alpha}, \bar{\omega}_{\alpha}\right]\right),
$$

where $\bar{\rho}_{\alpha}$ and $\bar{\omega}_{\alpha}$ are the solutions of the saddle point equations:

$$
\left.\frac{\delta \mathcal{H}\left[\nu_{\alpha}, \rho_{\alpha}, \omega_{\alpha}\right]}{\delta \rho_{\mathbf{k}, \alpha}}\right|_{\left(\bar{\rho}_{\alpha}, \bar{\omega}_{\alpha}\right)}=\left.\frac{\delta \mathcal{H}\left[\nu_{\alpha}, \rho_{\alpha}, \omega_{\alpha}\right]}{\delta \omega_{\mathbf{k}, \alpha}}\right|_{\left(\bar{\rho}_{\alpha}, \bar{\omega}_{\alpha}\right)}=0
$$


We obtain for $\bar{\rho}_{\alpha}$ and $\bar{\omega}_{\alpha}$

$$
\bar{\rho}_{\alpha}=\rho_{H S}^{\alpha}\left[\bar{\nu}_{\alpha}-\mathrm{i} \bar{\omega}_{\alpha}\right], \quad \bar{\omega}_{\alpha}=-\mathrm{i}\left[\bar{\rho}_{\alpha} \tilde{\Phi}_{\alpha \alpha}(0)+\sum_{\beta(\neq \alpha)} \bar{\rho}_{\beta} \tilde{\Phi}_{\alpha \beta}(0)\right]
$$

and $\rho_{H S}^{\alpha}\left[\bar{\nu}_{\alpha}-\mathrm{i} \bar{\omega}_{\alpha}\right]$ denotes the number density of the $\alpha$ th species of the hard sphere fluid with chemical potential $\bar{\nu}_{\alpha}-\mathrm{i} \bar{\omega}_{\alpha}$.

Substituting (12) in (11) we obtain

$$
\Xi_{M F}=\exp \left[\frac{\beta}{2} \sum_{\alpha, \beta} \rho_{H S}^{\alpha} \rho_{H S}^{\beta} \tilde{\phi}_{\alpha \beta}(0)\right] \Xi_{H S}\left[\bar{\nu}_{\alpha} ;-\mathrm{i} \bar{\omega}_{\alpha}\right] .
$$

Taking into account fluctuations. In order to take into account fluctuations we present CVs $\rho_{\mathbf{k}, \alpha}$ and $\omega_{\mathbf{k}, \alpha}$ in the form:

$$
\rho_{\mathbf{k}, \alpha}=\bar{\rho}_{\alpha} \delta_{\mathbf{k}}+\delta \rho_{\mathbf{k}, \alpha}, \quad \omega_{\mathbf{k}, \alpha}=\bar{\omega}_{\alpha} \delta_{\mathbf{k}}+\delta \omega_{\mathbf{k}, \alpha}
$$

where the quantities with a bar are given by (12).

The function $\ln \Xi_{H S}\left[\bar{\nu}_{\alpha} ;-\mathrm{i} \omega_{\alpha}\right]$ in (7) can be presented in the form of the cumulant expansion

$$
\ln \Xi_{H S}[\ldots]=\sum_{n \geqslant 0} \frac{(-\mathrm{i})^{n}}{n !} \sum_{\alpha_{1}, \ldots, \alpha_{n}} \sum_{\mathbf{k}_{1}, \ldots, \mathbf{k}_{n}} \mathfrak{M}_{\alpha_{1} \ldots \alpha_{n}}\left(k_{1}, \ldots, k_{n}\right) \delta \omega_{\mathbf{k}_{1}, \alpha_{1}} \ldots \delta \omega_{\mathbf{k}_{n}, \alpha_{n}} \delta_{\mathbf{k}_{1}+\cdots+\mathbf{k}_{n}}
$$

where $\mathfrak{M}_{\alpha_{1} \ldots \alpha_{n}}\left(k_{1}, \ldots, k_{n}\right)$ is the $n$th cumulant which is defined by

$$
\mathfrak{M}_{\alpha_{1} \ldots \alpha_{n}}\left(k_{1}, \ldots, k_{n}\right)=\left.\frac{\partial^{n} \ln \Xi_{H S}[\ldots]}{\partial \delta \omega_{\mathbf{k}_{1}, \alpha_{1}} \ldots \partial \delta \omega_{\mathbf{k}_{n}, \alpha_{n}}}\right|_{\delta \omega_{\mathbf{k}_{i}, \alpha_{i}}=0} .
$$

After the integration in (6) over $\delta \omega_{\mathbf{k}_{i}, \alpha_{i}}$ we obtain for $\Xi\left[\nu_{\alpha}\right]$

$$
\Xi\left[\nu_{\alpha}\right]=\Xi_{M F} \Xi^{\prime} \int(\mathrm{d} \delta \rho) \exp \left\{-\frac{1}{2 !} \sum_{\alpha, \beta} \sum_{\mathbf{k}} L_{\alpha \beta}(k) \delta \rho_{\mathbf{k}, \alpha} \delta \rho_{-\mathbf{k}, \beta}+\sum_{n \geqslant 3} \mathcal{H}_{n}\left(\delta \rho_{\alpha}\right)\right\} .
$$

Gaussian approximation. In the Gaussian approximation, which corresponds to taking into account in (15) only the terms with $n \leqslant 2\left(\mathcal{H}_{n} \equiv 0\right)$, we have $L_{\alpha \beta}(k)=C_{\alpha \beta}(k)$, where $C_{\alpha \beta}(k)$ are the Fourier transforms of the partial direct correlation functions. After integrating in (15) we arrive at the GPF of a $m$-component system in the random phase approximation (RPA).

\subsection{A two-component system of charged particles}

Let us consider now in more detail the particular case of a two-component system consisting of $N_{1}$ particles of species 1 and $N_{2}$ particles of species 2 . The particles of the species $\alpha=1$ are characterized by their hard sphere diameter $\sigma_{11}$ and their electrostatic charge $+q$ and those of species $\alpha=2$, characterized by diameter $\sigma_{22}$, bear opposite charge $-z q$. It is worth noting that such a model is of particular interest from the point of view of the phase transitions study in real ionic fluids.

For the model, equations (6) and (7) can be presented in the form:

$$
\Xi\left[\nu_{\alpha}\right]=\int(\mathrm{d} \rho)(\mathrm{d} Q)(\mathrm{d} \omega)(\mathrm{d} \gamma) \exp \left(-\mathcal{H}\left[\nu_{\alpha}, \rho, Q, \omega, \gamma\right]\right),
$$

and

$$
\begin{aligned}
\mathcal{H}\left[\nu_{\alpha}, \rho, Q, \omega, \gamma\right]= & \frac{1}{2} \sum_{\mathbf{k}}\left[\tilde{\Phi}_{N N}(k) \rho_{\mathbf{k}} \rho_{-\mathbf{k}}+\tilde{\Phi}_{Q Q}(k) Q_{\mathbf{k}} Q_{-\mathbf{k}}+2 \tilde{\Phi}_{N Q}(k)\right. \\
& \left.\times \rho_{\mathbf{k}} Q_{-\mathbf{k}}\right]-\mathrm{i} \sum_{\mathbf{k}}\left(\omega_{\mathbf{k}} \rho_{\mathbf{k}}+\gamma_{\mathbf{k}} Q_{\mathbf{k}}\right)-\ln \Xi_{H S}\left[\bar{\nu}_{\alpha} ;-\mathrm{i} \omega,-\mathrm{i} q_{\alpha} \gamma\right]
\end{aligned}
$$


where CVs $\rho_{\mathbf{k}}$ and $Q_{\mathbf{k}}$ describe fluctuations of the total number density and charge density, respectively

$$
\rho_{\mathbf{k}}=\sum_{\alpha} \rho_{\mathbf{k}, \alpha}, \quad Q_{\mathbf{k}}=\sum_{\alpha} q_{\alpha} \rho_{\mathbf{k}, \alpha} .
$$

For $\tilde{\Phi}_{A B}(k)(A, B=N, Q)$ we have the following expressions

$$
\begin{aligned}
\tilde{\Phi}_{N N}(k) & =\frac{1}{(1+z)^{2}}\left[z^{2} \tilde{\Phi}_{11}^{\mathrm{SR}}(k)+2 z \tilde{\Phi}_{12}^{\mathrm{SR}}(k)+\tilde{\Phi}_{22}^{\mathrm{SR}}(k)\right], \\
\tilde{\Phi}_{Q Q}(k) & =\frac{1}{(1+z)^{2}}\left[\tilde{\Phi}_{11}^{\mathrm{SR}}(k)-2 \tilde{\Phi}_{12}^{\mathrm{SR}}(k)+\tilde{\Phi}_{22}^{\mathrm{SR}}(k)\right]+\tilde{\Phi}^{\mathrm{C}}(k), \\
\tilde{\Phi}_{N Q}(k) & =\frac{1}{(1+z)^{2}}\left[z \tilde{\Phi}_{11}^{\mathrm{SR}}(k)+(1-z) \tilde{\Phi}_{12}^{\mathrm{SR}}(k)-\tilde{\Phi}_{22}^{\mathrm{SR}}(k)\right] .
\end{aligned}
$$

$\Xi_{H S}\left[\bar{\nu}_{\alpha} ;-\mathrm{i} \omega,-\mathrm{i} q_{\alpha} \gamma\right]$ is the GPF of a two-component system of the bare hard spheres with the renormalized chemical potential

$$
\bar{\nu}_{\alpha}=\nu_{\alpha}+\frac{1}{2} \sum_{\mathbf{k}} \tilde{\Phi}_{\alpha \alpha}^{\mathrm{SR}}(k)+\frac{q_{\alpha}^{2}}{2} \sum_{\mathbf{k}} \tilde{\Phi}^{\mathrm{C}}(k)
$$

in the presence of the local field

$$
\psi_{\alpha}\left(r_{i}\right)=-\mathrm{i} \omega\left(r_{i}\right)-\mathrm{i} q_{\alpha} \gamma\left(r_{i}\right),
$$

where $\omega=\left(z \omega_{1}+\omega_{2}\right) /(1+z)$ and $\gamma=\left(\omega_{1}-\omega_{2}\right) / q(1+z)$.

In this case the saddle point equations yield

$$
\bar{\rho}=\rho_{H S}\left[\bar{\nu}_{\alpha} ;-\mathrm{i} \bar{\omega},-\mathrm{i} q_{\alpha} \bar{\gamma}\right], \quad \bar{Q}=0, \quad \bar{\omega}=-\mathrm{i} \bar{\rho} \tilde{\Phi}_{N N}(0), \quad \bar{\gamma}=-\mathrm{i} \bar{\rho} \tilde{\Phi}_{N Q}(0)
$$

and we obtain for the GPF in the mean field approximation

$$
\Xi_{M F}=\exp \left[\frac{\beta}{2} \rho_{H S}^{2} \tilde{\Phi}_{N N}(0)\right] \Xi_{H S}\left[\bar{\nu}_{\alpha} ;-\mathrm{i} \bar{\omega},-\mathrm{i} q_{\alpha} \bar{\gamma}\right] .
$$

The cumulant expansion for $\ln \Xi_{H S}[\ldots]$ in terms of variables $\delta \gamma_{\mathbf{k}}$ and $\delta \omega_{\mathbf{k}}$ have the form:

$$
\ln \Xi_{H S}[\ldots]=\sum_{n \geqslant 0} \frac{(-\mathrm{i})^{n}}{n !} \sum_{i_{n} \geqslant 0} \sum_{\mathbf{k}_{1}, \ldots, \mathbf{k}_{n}} \mathfrak{M}_{n}^{\left(i_{n}\right)}\left(k_{1}, \ldots, k_{n}\right) \delta \gamma_{\mathbf{k}_{1}} \ldots \delta \gamma_{\mathbf{k}_{i_{n}}} \delta \omega_{\mathbf{k}_{i_{n+1}}} \ldots \delta \omega_{\mathbf{k}_{n}} \delta_{\mathbf{k}_{1}+\ldots+\mathbf{k}_{n}}
$$

where the $n$th cumulant is defined by

$$
\mathfrak{M}_{n}^{\left(i_{n}\right)}\left(k_{1}, \ldots, k_{n}\right)=\left.\frac{\partial^{n} \ln \Xi_{H S}[\ldots]}{\partial \delta \gamma_{\mathbf{k}_{1}} \ldots \partial \delta \gamma_{\mathbf{k}_{i_{n}}} \partial \delta \omega_{\mathbf{k}_{i_{n+1}}} \ldots \partial \delta \omega_{\mathbf{k}_{n}}}\right|_{\delta \gamma_{\mathbf{k}}=0, \delta \omega_{\mathbf{k}}=0}
$$

and index $i_{n}$ is used to indicate the number of variables $\delta \gamma_{\mathbf{k}}$ in the cumulant expansion.

As was shown [14], the free energy obtained in the RPA for such a model is independent of the charge asymmetry factor $z$. Therefore, the study of the effect of the charge asymmetry on the phase behaviour requires taking into account the cumulants of the order higher than the second order. In this relation it is important to have got the explicit expressions for the cumulants with $n \geqslant 2$. The next section is devoted to this issue.

\section{Reference system}

\subsection{A $m$-component mixture of "colour" hard spheres}

Let us consider a general case of a $m$-component inhomogeneous system of hard spheres with the local chemical potential $\nu_{\alpha}^{*}(r)$. We start with expression (8) under conditions that all the hard 
spheres have the same diameter $\sigma$ and $\nu_{\alpha}^{*}(r)=\bar{\nu}_{\alpha}+\psi_{\alpha}(r)$. In this case (8) can be presented in the form:

$$
\Xi_{H S}\left[Z^{*}\right]=\sum_{N \geqslant 0} \frac{1}{N !} \int \mathrm{d} \mathbf{r}_{1} \mathrm{~d} \mathbf{r}_{2} \ldots \mathrm{d} \mathbf{r}_{N} \prod_{j=1}^{N} Z^{*}\left(r_{j}\right) \exp \left[-\frac{\beta}{2} \sum_{i, j}^{N} \phi^{\mathrm{HS}}\left(r_{i j}\right)\right],
$$

where

$$
Z^{*}\left(r_{j}\right)=\sum_{\alpha} Z_{\alpha}^{*}\left(r_{j}\right)
$$

and

$$
Z_{\alpha}^{*}\left(r_{j}\right)=\exp \nu_{\alpha}^{*}\left(r_{j}\right)=\bar{Z}_{\alpha} \exp \left(-\mathrm{i} \omega_{\alpha}\left(r_{j}\right)\right), \quad \bar{Z}_{\alpha}=\exp \left(\bar{\nu}_{\alpha}\right) .
$$

Our goal is to derive explicit expressions for the cumulants $\mathfrak{M}_{\alpha_{1} \ldots \alpha_{n}}(1,2, \ldots, n)$ defined by

$$
\mathfrak{M}_{\alpha_{1} \ldots \alpha_{n}}(1,2, \ldots, n)=\left.\frac{1}{(-\mathrm{i})^{n}} \frac{\delta^{n} \ln \Xi_{H S}\left[Z^{*}\right]}{\delta \omega_{\alpha_{1}}(1) \delta \omega_{\alpha_{2}}(2) \ldots \delta \omega_{\alpha_{n}}(n)}\right|_{\delta \omega_{\alpha_{i}}(i)=0},
$$

where, for notational convenience, a position vector $\mathbf{r}_{i}$ is presented simply by $i$.

First we start with some important relations. We introduce the density distribution function $\rho_{n}(1,2, \ldots, n)$ and the correlation function $\rho_{n}^{T}(1,2, \ldots, n)[15]$ :

$$
\begin{aligned}
& \frac{\rho_{n}(1,2, \ldots, n)}{Z^{*}(1) Z^{*}(2) \ldots Z^{*}(n)}=\frac{1}{\Xi} \frac{\delta^{n} \Xi}{\delta Z^{*}(1) \delta Z^{*}(2) \ldots \delta Z^{*}(n)}, \\
& \frac{\rho_{n}^{T}(1,2, \ldots, n)}{Z^{*}(1) Z^{*}(2) \ldots Z^{*}(n)}=\frac{1}{\Xi} \frac{\delta^{n} \ln \Xi}{\delta Z^{*}(1) \delta Z^{*}(2) \ldots \delta Z^{*}(n)} .
\end{aligned}
$$

We also have the relation for the function $h_{n}(1,2, \ldots, n)$

$$
h_{n}(1,2, \ldots, n)=\frac{\rho_{n}^{T}(1,2, \ldots, n)}{\rho_{1}(1) \rho_{1}(2) \ldots \rho_{n}(1)} .
$$

Expressions (28)-(30) can be easily generalized for the case of a $m$-component mixture replacing $\rho_{n}(1,2, \ldots, n)$ by $\rho_{\alpha_{1} \ldots \alpha_{n}}(1,2, \ldots, n)$ and $\rho_{n}^{T}(1,2, \ldots, n)$ by $\rho_{\alpha_{1} \ldots \alpha_{n}}^{T}(1,2, \ldots, n)$, etc. As a result, we obtain for $\mathfrak{M}_{\alpha_{1}}(1)$

$$
\mathfrak{M}_{\alpha_{1}}(1)=\rho_{1}^{T}(1) \frac{Z_{\alpha_{1}}^{*}}{Z^{*}(1)}=\rho_{\alpha_{1}}(1),
$$

where we take into account that

$$
\rho_{1}^{T}(i)=\rho_{1}(i), \quad \frac{Z_{\alpha_{i}}^{*}}{Z^{*}(i)}=\frac{\rho_{\alpha_{i}}(i)}{\rho_{1}(i)}, \quad \rho_{1}(i)=\sum_{\alpha} \rho_{\alpha}(i) .
$$

As is readily seen, the first expression follows from (28)-(29) and the second one follows from (24) $-(25)$.

The higher order cumulants (up to $n=4$ ) can be written as

$$
\begin{aligned}
\mathfrak{M}_{\alpha_{1} \alpha_{2}}(1,2)= & \rho_{\alpha_{1}}(1)\left[\rho_{\alpha_{2}}(2) h_{2}(1,2)+\delta_{\alpha_{1} \alpha_{2}} \delta(1,2)\right], \\
\mathfrak{M}_{\alpha_{1} \alpha_{2} \alpha_{3}}(1,2,3)= & \rho_{\alpha_{1}}(1)\left[\rho_{\alpha_{2}}(2) \rho_{\alpha_{3}}(3) h_{3}(1,2,3)+\rho_{\alpha_{2}}(2) h_{2}(1,2) \delta_{\alpha_{2} \alpha_{3}} \delta(2,3)\right. \\
& +\rho_{\alpha_{2}}(2) h_{2}(1,2) \delta_{\alpha_{1} \alpha_{3}} \delta(1,3)+\rho_{\alpha_{3}}(3) h_{2}(1,3) \delta_{\alpha_{1} \alpha_{2}} \delta(1,2) \\
& \left.+\delta_{\alpha_{1} \alpha_{2}} \delta_{\alpha_{1} \alpha_{3}} \delta(1,2) \delta(1,3)\right],
\end{aligned}
$$




$$
\begin{aligned}
\mathfrak{M}_{\alpha_{1} \alpha_{2} \alpha_{3} \alpha_{4}}(1,2,3,4)= & \rho_{\alpha_{1}}(1)\left[\rho_{\alpha_{2}}(2) \rho_{\alpha_{3}}(3) \rho_{\alpha_{4}}(4) h_{4}(1,2,3,4)+3 \rho_{\alpha_{2}}(2) \rho_{\alpha_{3}}(3)\right. \\
& \times h_{3}(1,2,3)\left(\delta_{\alpha_{3} \alpha_{4}} \delta(3,4)+\delta_{\alpha_{2} \alpha_{4}} \delta(2,4)+\delta_{\alpha_{1} \alpha_{4}} \delta(1,4)\right) \\
& +2 \rho_{\alpha_{2}}(2) \rho_{\alpha_{4}}(4) h_{3}(1,2,4)\left(\delta_{\alpha_{2} \alpha_{3}} \delta(2,3)+\delta_{\alpha_{1} \alpha_{3}} \delta(1,3)\right) \\
& +\rho_{\alpha_{3}}(3) \rho_{\alpha_{4}}(4) h_{3}(1,3,4) \delta_{\alpha_{1} \alpha_{2}} \delta(1,2)+4 \rho_{\alpha_{2}}(2) h_{2}(1,2) \\
& \times\left(\delta_{\alpha_{2} \alpha_{3}} \delta_{\alpha_{2} \alpha_{4}} \delta(2,3) \delta(2,4)+\delta_{\alpha_{2} \alpha_{3}} \delta_{\alpha_{1} \alpha_{4}} \delta(2,3) \delta(1,4)\right. \\
& \left.+\delta_{\alpha_{2} \alpha_{4}} \delta_{\alpha_{1} \alpha_{3}} \delta(2,4) \delta(1,3)+\delta_{\alpha_{1} \alpha_{3}} \delta_{\alpha_{1} \alpha_{4}} \delta(1,3) \delta(1,4)\right) \\
& +2 \rho_{\alpha_{3}}(3) h_{2}(1,3)\left(\delta_{\alpha_{3} \alpha_{4}} \delta_{\alpha_{1} \alpha_{2}} \delta(3,4) \delta(1,2)+\delta_{\alpha_{1} \alpha_{4}} \delta_{\alpha_{1} \alpha_{2}}\right. \\
& \times \delta(1,4) \delta(1,2))+\rho_{\alpha_{4}}(4) h_{2}(1,4) \delta_{\alpha_{1} \alpha_{3}} \delta_{\alpha_{1} \alpha_{2}} \delta(1,3) \delta(1,2) \\
& \left.+\delta_{\alpha_{1} \alpha_{2}} \delta_{\alpha_{1} \alpha_{3}} \delta_{\alpha_{1} \alpha_{4}} \delta(1,2) \delta(1,3) \delta(1,4)\right] .
\end{aligned}
$$

In $(32)-(34) h_{n}(1,2, \ldots, n)$ is the correlation function of a one-component hard sphere system with $Z^{*}(i)$ given by $(25)$.

Formulas (32)-(34) are valid for an inhomogeneous system of equal diameter hard spheres in the external local field. Expressions (32)-(33) are of the same form as those recently obtained within the framework of the statistical field theory based on the Hubbard-Stratonovich transformation [16] (see (7.8) in [16]). Expression (34) is important in context of applying the approach proposed here for the study of phase transitions.

For a homogeneous system $\rho_{\alpha}(i)=\rho_{\alpha}$, where $\rho_{\alpha}$ is the number density of hard spheres of the $\alpha$ th species. In the case of model (1) describing a system of charged particles constrained by the condition of global electroneutrality, the expressions for the cumulants $\mathfrak{M}_{\alpha_{1} \alpha_{2}, \ldots, \alpha_{n}}(1,2, \ldots, n)$ should be supplemented by the two relations for partial densities $\rho_{\alpha}$, namely

$$
\sum_{\alpha} c_{\alpha}=1, \quad \sum_{\alpha} q_{\alpha} c_{\alpha}=0, \quad c_{\alpha}=\rho_{\alpha} / \rho
$$

\subsection{Two-component system of charged hard spheres}

Let us consider the particular case of a two-component model of charged hard spheres of the same diameter with the local chemical potential $\nu_{\alpha}^{*}(i)=\bar{\nu}_{\alpha}+\psi_{\alpha}(i)$, where $\bar{\nu}_{\alpha}$ and $\psi_{\alpha}$ are given by (19)-(20). First we consider cumulants $\mathfrak{M}_{n}^{\left(i_{n}\right)}(1, \ldots, n)$ (see (23)) for the case of an inhomogeneous system.

It is convenient to present $\mathfrak{M}_{n}^{\left(i_{n}\right)}(1, \ldots, n)$ in terms of the truncated (or connected) correlation functions $G_{n}(1,2, \ldots, n)$ defined by $[15]$

$$
G_{n}(1,2, \ldots, n)=\frac{\delta^{n} \log \Xi}{\delta \nu^{*}(1) \delta \nu^{*}(2) \ldots \delta \nu^{*}(n)} .
$$

As a result, we obtain the following expressions for the cumulants of the first order

$$
\mathfrak{M}_{1}^{(0)}(1)=G_{1}(1), \quad \mathfrak{M}_{1}^{(1)}(1)=q_{\alpha} c_{\alpha}(1) G_{1}(1),
$$

where $c_{\alpha}(i)=\rho_{\alpha}(i) / \rho(i)$ and summation over repeated indices is meant. For the cumulants of the second order we get

$$
\begin{aligned}
& \mathfrak{M}_{2}^{(0)}(1,2)=G_{2}(1,2), \quad \mathfrak{M}_{2}^{(1)}(1,2)=q_{\alpha} c_{\alpha}(2) G_{2}(1,2) \\
& \mathfrak{M}_{2}^{(2)}(1,2)=q_{\alpha}^{2} c_{\alpha}(1) G_{1}(1) \delta(1,2)+q_{\alpha} c_{\alpha}(1) q_{\beta} c_{\beta}(2)\left[G_{2}(1,2)-G_{1}(1) \delta(1,2)\right] .
\end{aligned}
$$

$\mathfrak{M}_{3}^{\left(i_{n}\right)}(1,2,3)$ with $i_{n}=0,1,2,3$ have the form:

$$
\mathfrak{M}_{3}^{(0)}(1,2,3)=G_{3}(1,2,3), \quad \mathfrak{M}_{3}^{(1)}(1,2,3)=q_{\alpha} c_{\alpha}(1) G_{3}(1,2,3),
$$




$$
\begin{aligned}
\mathfrak{M}_{3}^{(2)}(1,2,3)= & q_{\alpha} c_{\alpha}(1) q_{\beta} c_{\beta}(2)\left[G_{3}(1,2,3)-G_{2}(2,3) \delta(1,3)\right] \\
& +q_{\alpha}^{2} c_{\alpha}(1) G_{2}(2,3) \delta(1,3) \\
\mathfrak{M}_{3}^{(3)}(1,2,3)= & q_{\alpha} c_{\alpha}(1) q_{\beta} c_{\beta}(2) q_{\gamma} c_{\gamma}(3)\left[G_{3}(1,2,3)-3 G_{2}(1,2) \delta(2,3)\right. \\
& \left.+2 G_{1}(1) \delta(1,3) \delta(2,3)\right]+3 q_{\alpha}^{2} c_{\alpha}(1) q_{\beta} c_{\beta}(2)\left[G_{2}(1,2)\right. \\
& \left.-G_{1}(1) \delta(1,2)\right] \delta(1,3)+q_{\alpha}^{3} c_{\alpha}(1) G_{1}(1) \delta(1,2) \delta(2,3) .
\end{aligned}
$$

Finally, we obtain for $\mathfrak{M}_{4}^{\left(i_{n}\right)}(1,2,3,4)$

$$
\begin{aligned}
\mathfrak{M}_{4}^{(0)}(1,2,3,4)= & G_{4}(1,2,3,4), \quad \mathfrak{M}_{4}^{(1)}(1,2,3,4)=q_{\alpha} c_{\alpha}(1) G_{4}(1,2,3,4) \\
\mathfrak{M}_{4}^{(2)}(1,2,3,4)= & q_{\alpha} c_{\alpha}(1) q_{\beta} c_{\beta}(2)\left[G_{4}(1,2,3,4)-G_{3}(2,3,4) \delta(1,4)\right] \\
& +q_{\alpha}^{2} c_{\alpha}(1) G_{3}(2,3,4) \delta(1,2), \\
\mathfrak{M}_{4}^{(3)}(1,2,3,4)= & q_{\alpha} c_{\alpha}(1) q_{\beta} c_{\beta}(2) q_{\gamma} c_{\gamma}(3)\left[G_{4}(1,2,3,4)-3 G_{3}(2,3,4) \delta(1,4)\right. \\
& \left.+2 G_{2}(3,4) \delta(1,4) \delta(2,3)\right]+3 q_{\alpha}^{2} c_{\alpha}(1) q_{\beta} c_{\beta}(2)\left[G_{3}(2,3,4)\right. \\
& \left.-G_{2}(3,4) \delta(2,4)\right] \delta(1,3)+q_{\alpha}^{3} c_{\alpha}(1) G_{2}(3,4) \delta(1,2) \delta(2,3) \\
\mathfrak{M}_{4}^{(4)}(1,2,3,4)= & q_{\alpha} c_{\alpha}(1) q_{\beta} c_{\beta}(2) q_{\gamma} c_{\gamma}(3) q_{\zeta} c_{\zeta}(4)\left[G_{4}(1,2,3,4)-6 G_{3}(1,2,3) \delta(1,4)\right. \\
& \left.+11 G_{2}(1,2) \delta(2,3) \delta(1,4)-6 G_{1}(1) \delta(2,3) \delta(1,4) \delta(1,2)\right] \\
& +6 q_{\alpha}^{2} c_{\alpha}(1) q_{\beta} c_{\beta}(2) q_{\gamma} c_{\gamma}(3) G_{3}(2,3,4) \delta(1,4)+4 q_{\alpha}^{3} c_{\alpha}(1) q_{\beta} c_{\beta}(2) \\
& \times\left[G_{2}(2,3)-G_{1}(2) \delta(2,3)\right] \delta(1,3) \delta(3,4)+3 q_{\alpha}^{2} c_{\alpha}(1) q_{\beta}^{2} c_{\beta}(2) \\
& \times\left[G_{2}(2,3)-G_{1}(2) \delta(2,3)\right] \delta(1,3) \delta(2,4)+q_{\alpha}^{4} c_{\alpha}(4) G_{1}(1) \\
& \times \delta(1,2) \delta(2,3) \delta(3,4) .
\end{aligned}
$$

Homogeneous case For the homogeneous system, $c_{\alpha}(i)=c_{\alpha}$ where $c_{\alpha}$ is the concentration of the $\alpha$ th species. In this case expressions (37)-(45) reduce due to the electroneutrality condition. As a result, we get the following recurrence formulas for the cumulants in the Fourier space representation

$$
\begin{aligned}
\mathfrak{M}_{n}^{(0)}\left(\mathbf{k}_{1}, \mathbf{k}_{2}, \ldots, \mathbf{k}_{n}\right)= & \widetilde{G}_{n}\left(\mathbf{k}_{1}, \mathbf{k}_{2}, \ldots, \mathbf{k}_{n}\right) \\
\mathfrak{M}_{n}^{(1)}\left(\mathbf{k}_{1}, \mathbf{k}_{2}, \ldots, \mathbf{k}_{n}\right)= & 0 \\
\mathfrak{M}_{n}^{(2)}\left(\mathbf{k}_{1}, \mathbf{k}_{2}, \ldots, \mathbf{k}_{n}\right)= & q_{\alpha}^{2} c_{\alpha} \widetilde{G}_{n-1}\left(\mathbf{k}_{1}, \mathbf{k}_{2}, \ldots, \mathbf{k}_{n-1}+\mathbf{k}_{n}\right) \\
\mathfrak{M}_{n}^{(3)}\left(\mathbf{k}_{1}, \mathbf{k}_{2}, \ldots, \mathbf{k}_{n}\right)= & q_{\alpha}^{3} c_{\alpha} \widetilde{G}_{n-2}\left(\mathbf{k}_{1}, \mathbf{k}_{2}, \ldots, \mathbf{k}_{n-2}+\mathbf{k}_{n-1}+\mathbf{k}_{n}\right) \\
\mathfrak{M}_{n}^{(4)}\left(\mathbf{k}_{1}, \mathbf{k}_{2}, \ldots, \mathbf{k}_{n}\right)= & 3\left[q_{\alpha}^{2} c_{\alpha}\right]^{2} \widetilde{G}_{n-2}\left(\mathbf{k}_{1}, \mathbf{k}_{2}, \ldots, \mathbf{k}_{n-2}+\mathbf{k}_{n-1}+\mathbf{k}_{n}\right)+\left(q_{\alpha}^{4} c_{\alpha}\right. \\
& \left.-3\left[q_{\alpha}^{2} c_{\alpha}\right]^{2}\right) \widetilde{G}_{n-3}\left(\mathbf{k}_{1}, \mathbf{k}_{2}, \ldots, \mathbf{k}_{n-3}+\ldots+\mathbf{k}_{n}\right)
\end{aligned}
$$

where $\widetilde{G}_{n}\left(\mathbf{k}_{1}, \mathbf{k}_{2}, \ldots, \mathbf{k}_{n}\right)$ is the Fourier transform of the $n$-particle truncated correlation function of a one-component hard sphere system with the density $\bar{\rho}$ defined by (12).

It is worth noting that $\mathfrak{M}_{2}^{(2)}\left(\mathbf{k}_{1}, \mathbf{k}_{2}\right), \mathfrak{M}_{3}^{(3)}\left(\mathbf{k}_{1}, \mathbf{k}_{2}, \mathbf{k}_{3}\right)$ and $\mathfrak{M}_{4}^{(4)}\left(\mathbf{k}_{1}, \ldots, \mathbf{k}_{4}\right)$ can be reduced to the expressions obtained in [16] (see (7.8) in [16]).

For the case of the restricted primitive model (RPM), a charge symmetric model with $z=1$, the above expressions can be rewritten as

$$
\begin{array}{ll}
\mathfrak{M}_{n}^{(1)} \equiv 0, & \mathfrak{M}_{n}^{(2)}=q^{2} \widetilde{G}_{n-1} \\
\mathfrak{M}_{n}^{(3)} \equiv 0, & \mathfrak{M}_{n}^{(4)}=q^{4}\left(3 \widetilde{G}_{n-2}-2 \widetilde{G}_{n-3}\right)
\end{array}
$$


Putting $q=1$ in (47) we arrive at recurrence relations for the cumulants of a mixture of neutral hard spheres with concentration $\left.c_{\alpha}=1 / 2[17]\right)$.

\section{Conclusions}

Based on the method of CVs we obtain the exact expression for the functional of the GPF for a $m$-component model of an ionic fluid with size and charge asymmetry. The functional integral is written in terms of $\mathrm{CVs} \rho_{\mathbf{k}, \alpha}(\alpha=1,2, \ldots, m)$ describing the density fluctuations of the $\alpha$ th species. The expression includes the logarithm of the GPF of the reference system which can be presented as a cumulant expansion. We pay special attention to the correlation functions of the reference system. The reference system for the model under consideration is a $m$-component system of equal diameter hard spheres with the local chemical potentials $\nu_{\alpha}^{*}(r)$. We call such a model a system of "colour" hard spheres. We obtain explicit expressions for the partial cumulants, the partial $n$ particle truncated (connected) correlation functions $(n \leqslant 4)$ of an inhomogeneous "colour" hard sphere system.

We consider the particular case of a two-component model. Here we introduce the two sets of CVs: $\rho_{\mathbf{k}}$ and $Q_{\mathbf{k}}$ describing the fluctuations of the total number density and the charge density, respectively. We choose the reference system as a two-component hard sphere system of equal diameters with the local chemical potentials which include the charges and reflect the fact that the Coulomb interactions are available in the full system. For the homogeneous ionic model we derive the recurrence formulas which relate the cumulants of the initial two-component reference system with the truncated correlation functions of a one-component hard sphere system. The relations will be used in our study of the phase behaviour of an ionic fluid with the charge and size asymmetry. The results will be published elsewhere.

\section{Acknowledgement}

We thank J.-M. Caillol for the useful discussions. Part of this work was carried out within the framework of the cooperation project between the CNRS and the NASU (ref. CNRS).

\section{References}

1. Bohm D., Pines D., Phys. Rev., 1951, 82, 625.

2. Zubarev D.N., Dokl. Acad. Nauk SSSR, 1954, 95, 757 (in Russian).

3. Yukhnovsky I.R., Zh. Eksp. Ter. Fiz., 1958, 34, 379 (in Russian).

4. Yukhnovskii I.R. Phase Transitions of the Second Order: Collective Variables Method. Singapore, World Scientific, 1987.

5. Yukhnovskii I.R., Proceedings of the Steklov Institute of Mathematics, 1992, 2, 223.

6. Patsagan O.V., Yukhnovskii I.R., Teor. Mat. Fiz., 1990, 83, 72.

7. Yukhnovskii I.R., Patsahan O.V., J. Stat. Phys., 1995, 81, 647.

8. Stratonovich R.L., Sov. Phys. Solid State, 1958, 2, 1824.

9. Hubbard J., Phys. Rev. Lett., 1959, 2, 77.

10. Caillol J.-M., Patsahan O., Mryglod I., Physica A., 2006 (in press).

11. Yukhnovskii I.R., Holovko M.F. Statistical Theory of Classical Equilibrium Systems. Naukova Dumka, Kiev, 1980 (in Russian).

12. Hansen J.P., McDonald I.R. Theory of simple liquids. Academic Press, 1986.

13. Levitskii R.R., Sorokov S.I., Condens. Matter Phys., 1993, No. 1, 43.

14. Caillol J.-M., Mol. Phys., 2005, 103, 1271.

15. Stell G. In Phase Transitionss and Critical Phenomena, 5b, C. Domb and M.S. Green, eds., Academic Press, New York, 1975.

16. Caillol J.-M., J. Stat. Phys., 2004, 115, 1461.

17. Patsahan O.V., Condens. Matter Phys., 2004, 7, 35. 


\section{Функціональне представлення великої статистичної суми для багатокомпонентної системи заряджених частинок: кореляційні функції системи відліку}

\section{О.В.Пацаган, І.М.Мриглод}

Інститут фізики конденсованих систем НАН України, вул. Свєнціцького, 1, 79011 Львів

Отримано 27 лютого 2006 р., в остаточному вигляді - 29 червня 2006 р.

На основі методу колективних змінних з системою відліку, отримано точний вираз для функціоналу
великої статистичної суми $m$-компонентної іонної моделі з асиметрією розмірів та зарядів. Особли-
ва увага приділяється $n$-частинковим кореляційним функціям системи відліку, яка представляється
як $m$-компонентна система “кольорових" твердих сфер однакового діаметру. Детально розглянуто
випадок двокомпонентної моделі, для якої отримано рекурентні формули для кореляційних функцій.
Також проаналізовано випадок $m$-компонентної неоднорідної системи “кольорових" твердих сфер.

Ключові слова: функціональні методи, система відліку, метод колективних змінних, асиметрична іонна модель, система "кольорових" твердих сфер

PACS: $05.70 . F h, 05.70 . J k, 65.10 .+h$ 